\title{
Utilization of GPS surface reflected signals to provide aircraft altitude verification for SVS
}

\author{
George G. Ganoe, Steven D. Young \\ NASA Langley Research Center, Hampton, Va. 23681-2199
}

\begin{abstract}
The Global Positioning System (GPS) consists of a constellation of Earth orbiting satellites that transmit continuous electromagnetic signals to users on or near the Earth surface. At any moment of time, at least four GPS satellites, and sometimes nine or more, are visible from any point. The electromagnetic signal transmitted from the satellites is reflected to at least some degree from virtually every place on the Earth. When this signal is received by a specially constructed receiver, its characteristics can be used to determine information about the reflected surface. One piece of information collected is the time delay encountered by the reflected signal versus the direct signal. This time delay can be used to determine the altitude (or height) above the local terrain when the terrain in the reflection area is level. However, given the potential of simultaneously using multiple reflections, it should be possible to also determine the elevation above even terrains where the reflecting area is not level. Currently an effort is underway to develop the technology to characterize the reflected signal that is received by the GPS Surface Reflection Experiment (GSRE) instrument. Recent aircraft sorties have been flown to collect data that can be used to refine the technology. This paper provides an update on the status of the instrument development to enable determination of terrain proximity using the GPS Reflected signal. Results found in the data collected to date are also discussed.
\end{abstract}

\section{INTRODUCTION}

Since the advent of the Global Positioning System (GPS) which enabled navigation to high accuracy anywhere on the surface of the earth, and its nearby surroundings, many unforeseen applications of the microwave radio signals transmitted by the GPS satellites have emerged. A subset of these applications take advantage of an unwanted but unavoidable source of error for navigation that is caused by the reception of multipath due to reflections of the signal from some surfaces. This effect can be detected by flying aircraft with the appropriate sensing antennas and GPS receivers as the signals transmitted by the GPS satellites are reflected by the surface of the earth. This paper describes a method for measuring the characteristics of the reflected signals, and using that information to enable verification of Digital Elevation Models (DEM) used by Synthetic Vision Systems (SVS). Section two of the paper will provide an overview of SVS, giving a description of what it does, and compare and contrast it with existing aircraft safety systems. The overview shows how a reliable SVS could significantly improve the capabilities of aircraft to fly safely even when external visibility is impaired. Section three presents the link between the SVS and the need for a GPS-based reflectometer by introducing the concept of navigation system integrity, the problem of achieving the needed integrity for the SVS DEMs, and the role that a GPS-based reflectometer can play in solving this problem. Section four briefly reviews the background of GPS reflectometry by taking a look at some of the applications that have been developed to take advantage of the technique and also provides an introduction to the concepts that make GPS-based reflectometry a viable technique for helping to solve the problem of the SVS DEM integrity. Finally, section five provides an overview 
of the instrument development activities at NASA Langley Research Center for the GPS-based reflectometer that has supported many of the GPS surface reflection applications, the steps being taken to enhance the instrument in order to enable the desired DEM validation capability, and some information about expected measurement accuracy.

\section{SVS OVERVIEW}

SVS provides pilots with a virtual visual depiction of the external environment. By enabling pilots to maintain their sense of vision regardless of the weather conditions, SVS has the potential to improve safety-of-flight and operational efficiency. SVS depictions can be portrayed on almost any flight-qualified display media. Prototype implementations have used both head-down displays and head-up displays that provide aircraft state, guidance, and navigation information, along with a perspective view of external features ${ }^{1}$. In an SVS, the geographic location and dimensions of many of these features are stored in on-board databases; this is in contrast with an Enhanced Vision System (EVS). An EVS provides a display of the forward external scene through the use of imaging sensors.

The operational objective of SVS is to increase pilot situational awareness in low visibility conditions to a level of awareness similar to clear-weather flying. As such, SVS is being proposed as a mitigation strategy for accident categories such as Controlled-Flight-Into-Terrain (CFIT), runway incursions, and low-visibility-induced loss-of-control scenarios. SVS may also enable operational credits by allowing advanced precision approach procedures and lower minimums.

When serving as a Primary Flight Display (PFD), SVS presents stored geographic feature data and dynamic real-time data to pilots from an ego-centric perspective. The SVS concept is based on a presumption that the locations of most geographic features do not change frequently or significantly over time and therefore, can be stored on-board. The stored feature data can represent terrain, cultural features such as rivers and roads, obstacles such as buildings and towers, and navigation references such as omni-directional rotating beacon locations and airport reference points.

SVS displays also present data that does change significantly and frequently over time. This data must be obtained inflight from a sensor, a communication channel, or the crew. Dynamic data includes aircraft position and attitude, traffic locations, weather, air traffic control instructions, and flight information services such as Notices to Airmen (NOTAMs).

The displays are the visual components of the SVS that form the interface between the SVS computer system and the pilot. The SVS processor uses the required inputs to generate the information that is depicted on the displays. The SVS computer system interfaces with a database server: an onboard unit that stores and manages the static data required by SVS.

A closely related cockpit display application is the Terrain Awareness and Warning System (TAWS). TAWS implementations have been mandated by the Federal Aviation Administration (FAA) for nearly all aircraft to increase pilot's situational awareness with respect to terrain. Various TAWS systems have been developed that are very similar to SVS as both are based on the same premise - using feature databases instead of sensors to re-create the external environment. However, there are two major differences between TAWS and SVS. First, as the name implies, TAWS only provides information about the terrain environment. Second, TAWS is currently certified and approved operationally as an 'advisory-only' function. In essence, use of this regulatory term is a way of informing pilots that they should never use the TAWS information as a primary navigational aid (nav-aid) when making tactical steering 
decisions. On the other hand, SVS is being designed for applications that range from purely advisory to flight-critical. A flight-critical SVS function would allow pilots to make tactical steering inputs based solely on the SVS depiction. The implication of this operational objective is one of the primary drivers of this research and will be discussed further below.

\section{SVS INTEGRITY MONITORING AND GPS BI-STATIC RADAR TECHNOLOGY}

Navigation system integrity refers to the ability of the system to provide timely warnings to users when the system should not be used for navigation. With respect to SVS, the same definition holds only under the circumstances described previously -- SVS is being used as a primary nav-aid. For example, when guidance from other nav-aids is unavailable or of insufficient integrity, the SVS will be the navigation system that is used and thus, timely warnings must be provided to the pilot when the SVS should not be used for this purpose. The probability that the SVS will provide timely warnings indicating when it should not be used for its intended function is defined as SVS system integrity.

In the specific case of Digital Elevation Models (DEMs), data integrity is related to system integrity in that system integrity can be compromised when data errors exist in the DEM that result in misleading information that is not detected by the operational system. Three techniques to boost system integrity can be considered in the presence of DEM errors: fault-avoidance, fault-tolerance, and fault-detection.

With respect to geographic feature database errors, the fault-avoidance technique, also known as proof-of-correctness, focuses on validation. The designer may attempt to prove that there are no errors by checking all data, or attempt to prove that the data collection process cannot produce errors. Depending on the amount of data to be validated and/or the complexity of the collection process, this can become impractical.

Fault-tolerance approaches are used often in aviation to recover from hardware failures. With respect to geographic feature database errors, a safety assessment would be performed to determine the failure modes that would contribute to undesired top-level events, such as misleading information. The system can then be designed to eliminate or mitigate the effects of these failure modes. Architectural techniques such as system redundancy, perhaps even using dissimilar implementations, can increase system integrity.

Fault-detection methods are characterized by monitoring and/or built-in-test equipment functions that enable detection of system failures in real-time. The effect of monitoring is to lower the probability of undetected failure/error, which in turn will increase system integrity. The monitoring approach is the one that has been selected for this research based on the assumption that the geographic feature databases will contain errors that may provide misleading information under certain operational conditions, and that using the other two approaches may be impractical/infeasible.

As will be shown, without a monitoring function, SVS integrity will depend on the GPS integrity, the Inertial Navigation System (INS) integrity, and the inherent integrity of the geographic feature databases. While high levels of GPS integrity may be available via the receiver's internal monitor or an augmentation service and INS integrity may be provided by redundant IRUs, the integrity of DEMs has not been quantified and therefore, SVS integrity cannot be quantified. One objective of this research is to determine whether DEM integrity can be bounded using multiple independent measurements and real-time consistency checking. Specifically, the purpose of this study is to investigate the concept of using measurements from GPS Bi-static Radar (GPS-BR) receiver technology as a component of a 
Forward-Looking Autonomous Integrity Monitor (FLAIM). In Illustration 1 , the $T_{\mathrm{j}} \mathrm{s}$ represent test-statistics that represent agreement between metrics derived from on-board sensors and data bases for specific coverage regions. Using these agreement test-statistics, FLAIM is designed to provide a bounded level of integrity for geographic feature databases such as DEMs so that safe operational constraints can be specified ${ }^{2}$.

GPS-BR receiver technology is based on the notion of using bottom-mounted antennas to receive the satellite-based transmissions that have been reflected from the terrain (see

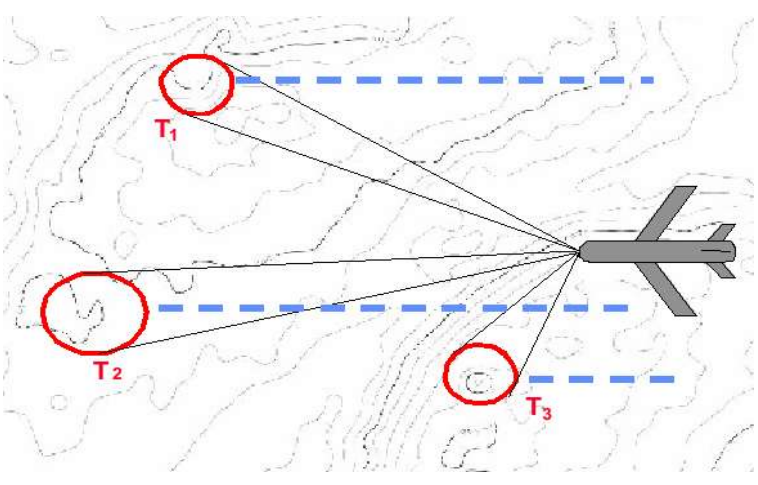

Illustration 1 - FLAIM concept depiction Illustration 2) ${ }^{3}$. This concept is still in an experimental stage but shows promise as a potential low-cost FLAIM sensor. Path delay has been suggested as an agreement statistic whereby one path delay is measured and another is synthesized using a DEM. The remainder of this paper describes one implementation of GPS-BR technology aimed at providing these two path delay metrics as well as altitude estimates. Altimetry is an important side benefit of GPS-BR technology particularly for those aircraft not equipped with SVS.

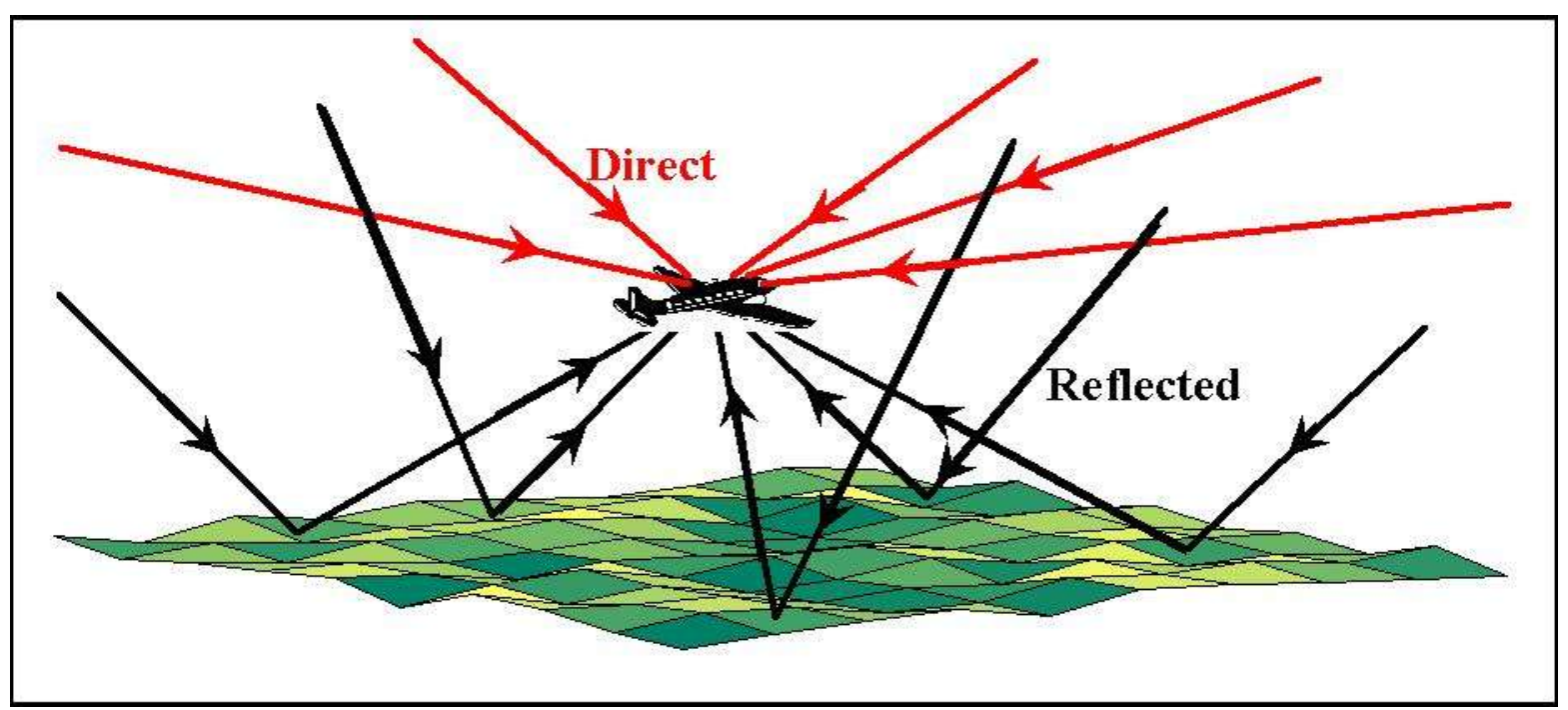

Illustration 2 - GPS signals reflected from terrain

\section{GPS REFLECTOMETRY BACKGROUND}

While the primary purpose of the GPS constellation is to provide a source for global navigation, it has been known since its inception that multipath effects would be a cause of error that required attention in the configuration of receiving systems. Since the initial characterization of the multipath at GPS frequencies by Auber, Bibaut, and Rigal ${ }^{4}$, the body of knowledge about the characteristics of the reflected GPS signal from various surfaces has proliferated, and includes applications such as retrieval of ocean surface wind speeds using GPS surface reflection data ${ }^{5}$, coastal area and wetlands mapping, ice sheet characterization, and soil moisture monitoring. Other applications that have been described or demonstrated using the reflected signal include altimetry over ocean and land flight paths, and determination of Ionospheric delay over the ocean from spacecraft. 
Since the GPS signal is reflected from the surface of the ocean, it has been proposed that the reflected signal would be detectable from low Earth orbiting satellite altitudes. Since such a reflected signal would be subjected to the delay from two passes through the Earth's atmosphere including the ionosphere, such a signal could be used to determine the Ionospheric delay over areas where it is not typically be measured directly ${ }^{6}$. This information could then be used to provide the Ionospheric correction for a satellite-based single frequency altimeter instrument.

It has been shown that ocean surface roughness due to the surface winds causes the reflecting surface area to increase relative to the roughness of the surface. This results in a spreading of the reflected signal received over time and a reduction in the instantaneous power received at any specific time. The resulting power versus delay characteristic can be predicted for given wind speeds, satellite positions, and aircraft flight regimes. The actual measurements from a GPS-BR receiver can be matched to these predictions to accurately estimate the surface wind speed at the time of the measurement ${ }^{7}$.

The amount of power in the reflected GPS signal varies depending on the characteristics of the reflecting surface. While water is almost perfectly reflective, wetland areas are less reflective, and the reflectivity of soil is dependent on the soil moisture and vegetative ground cover. These facts have been verified by performing measurements over wetland and coastal areas and then correlating the resulting data to coastline maps ${ }^{8}$.

A number of aircraft flights have been flown to record the reflected signal over various terrain types, and it has been noted that there are GPS signal reflections from virtually every type of surface. Ongoing research is being conducted to characterize the recorded data and correlate with terrain/surface characteristics.

Using the range coding information inherent in the characteristics of the GPS signal, it is possible to estimate the time difference in reception between the direct signal from a GPS satellite, and the same signal reflected from the surface. With the time difference, a knowledge of the relative positions of the aircraft and the GPS satellite, and an assumption that the reflecting surface is level, the altitude above the surface can be computed (see Illustration 3). Even when the reflecting surface is known not to be level, but characteristics of the terrain are known, the distance to the reflecting surface can still be computed.

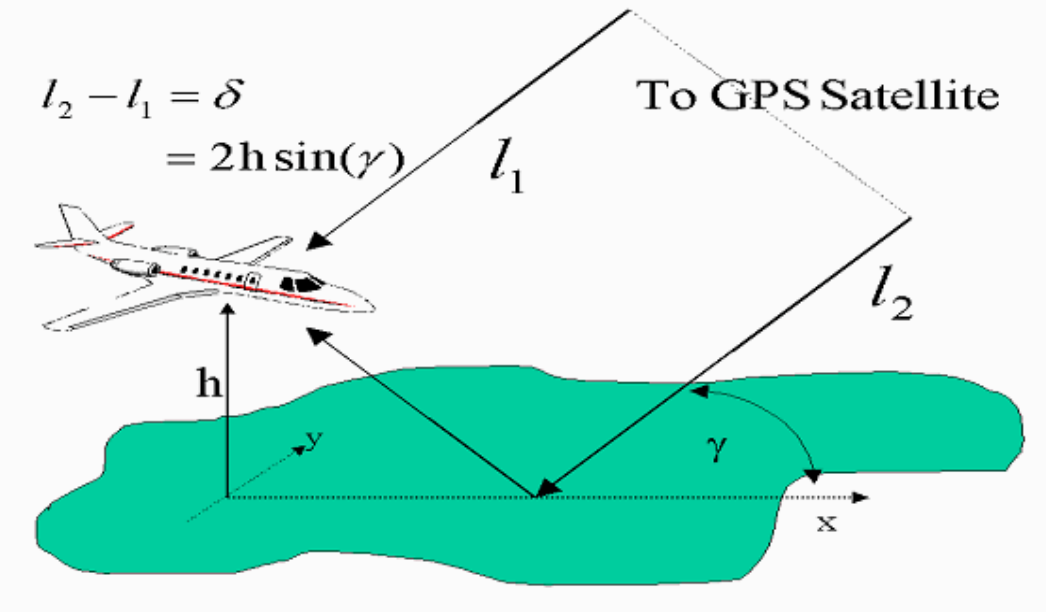

Illustration 3 - Height computation using reflected signal 
It is likely that over many terrains, for each GPS satellite signal there will be multiple reflections received, and those signals are spread over time. By selecting the appropriate satellite signal for monitoring the reflections, we can be assured that the nearest of the reflected signals is seen first in received power versus delay data sets. Assuming that the nearest reflected signal is seen first, the time difference between the direct and the reflected signal from that point can be estimated by detecting the onset of reception of the reflected signal. This is evidenced by the detection of an image of the direct signal distinctly discriminated from the signal background noise. Since the total reflected signal power is dependent on the type of terrain that the signal is being reflected from, the power versus delay characteristic must be analyzed over multiple range bins in order to estimate the actual time of onset of the reflected signal.

\section{GPS REFLECTOMETRY INSTRUMENT DEVELOPMENT}

The current GPS-based reflectometer instrument is a second generation compact version of the GPS Surface Reflection Experiment (GSRE) instrument originally demonstrated in $1997^{9}$. The original instrument consisted of an IBM PC-AT compatible computer system with a GEC Plessey dual RF front end GPS receiver development system installed into an ISA adapter slot. The system ran modified versions of the Plessey Development system software on the MS-DOS operating system. The modified versions of the software provided the capability for estimating the power versus delay for the reflected GPS signal using one of two methods. The initial method utilized a technique of pairing direct signals in receiver channels one through six with with reflected versions of each of the direct signals in channels seven through twelve respectively. The reflected channel signal measurement was stepped through a set of delays, offset from the actual signal time that the direct signal was received, where reflected signal information was expected, and the power level of the signal was recorded for each of the delay bins. This reflected signal measurement method had the advantage that reflections from up to six different satellites could be measured at the same time, and the use of six direct channels permitted the capability of getting a position solution while recording reflected data. The disadvantage of the stepping method is that the integration time for each delay bin was very short, and hence the signal to noise ratio (SNR) for the data was fairly low. In order to increase the SNR, another measurement technique was implemented that used one channel for the direct signal, and the other eleven channels to measure reflected signals for multiple delay bins in parallel. With eleven channels dedicated to receiving reflected signal information, 22 range bins, two per channel for the eleven reflected signal channels, could be collected in parallel. This resulted in a much greater SNR, and consequently much higher quality data. The disadvantage of this approach was that a second GPS receiver was required to track the position solution, and the GPS-BR receiver needed constant monitoring to insure that it was tracking a satellite on its direct channel. For the early versions of both tracking methods, the bulk delay that was needed to get to the beginning of the reflected signal information was entered manually. The bulk delay is a time delay between the time a signal is received at the direct channel and the time a corresponding signal is sampled in the first delay bin of the reflected signal channels. As the techniques matured, an algorithm for computing the bulk delay based on receiver height was incorporated into the software for the stepping code method. A reflected signal search and track algorithm was incorporated into the parallel software, since no position solution could be computed with only one direct channel.

The current version of the instrument consists of a PC-104 based Pentium system with a dual RF channel GPS receiver interfaced to the PC-104 bus using a custom designed interface (see Illustration 4). The software is derived from the original Plessey Development System software, but has now been extensively modified to provide additional recorded data. The primary technique used for data collection of the reflected signals is one derived from the parallel technique implemented in the original receiver. In order to ensure that a position solution can be computed, the first five channels of the twelve channel GPS receiver hardware are used to track direct satellite signals, and the other seven channels 
measure 14 delay bins of the reflected signal from one of the satellites being tracked in channels one through five. Using the computed position solution, the bulk delay is determined based on the receiver height, and the reflected channel delay bin positions are set automatically. The algorithm for determining the bulk delay based on position height was sufficient for ocean surface measurements, and for measurements over terrain for low elevations since these are close enough to the reference ellipsoid that the computed bulk delay is close enough that the reflected signal occurs within the range of bins that are available. However, in anticipation of the need to measure reflected signals over terrains where the elevation is significantly different from the reference, the bulk delay algorithm was modified to be offset based on information

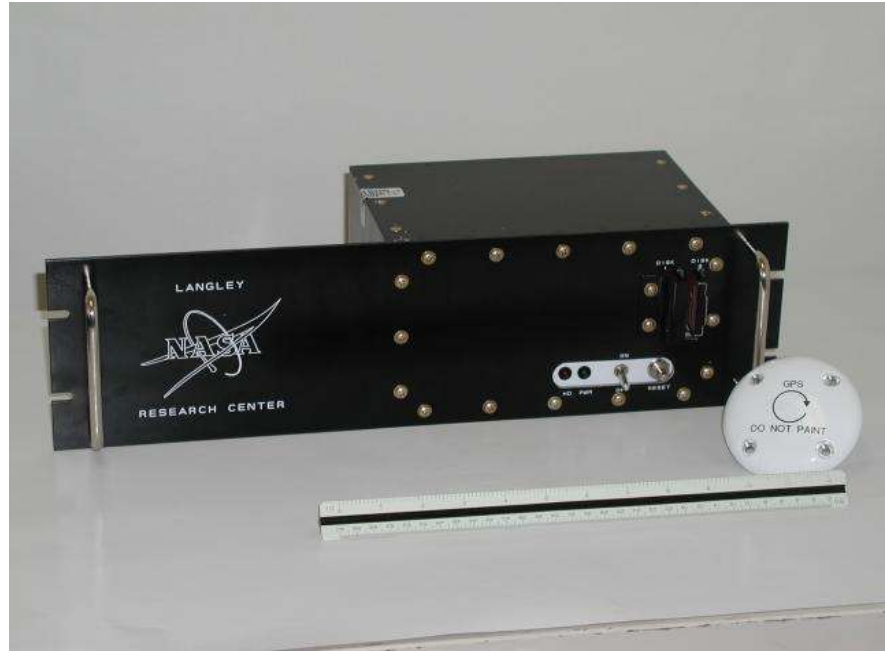

Illustration 4 - Current GPS Reflectometer in flight qualified package contained in a low resolution DEM which has been placed in the receiver's disk storage. This new algorithm has been recently validated, and as a result, the GPS-BR receiver can now be used over any area for which it has a low resolution DEM. Since the reflected signal is recorded over a range of delay bins, the accuracy of the DEM is not critical. So long as the actual elevation is within about 1000 meters of the expected elevation, the GPS-BR receiver (i. e. reflectometer) operates acceptably.

Another recent innovation in the operation of the GPS-BR receiver was implemented to enable the instrument to continuously select the most appropriate satellite from among the five direct tracking channels for the master channel used to control the reflected signal measurements. The instrument has historically used the first channel as the master, and the GPS satellite tracked on that channel would be the one used for measuring reflected signals regardless of the satellite's characteristics. This often lead to reflected data being recorded for low elevation satellites when more appropriate higher elevation satellites were available. The new software can automatically select the master channel from any of the five direct measurement channels such that individual reflected signal measurement arcs remain as long as possible while still maintaining measurements from relatively high elevation satellites. In order to enable the rapid switch of the reflected channels from one master to another, an algorithm was developed to provide rapid slewing of the code and carrier Digitally Controlled Oscillators (DCOs) for the reflected channels to the new values that they would need after the switch. A variation of this technique will likely prove valuable for use in the implementation of the surface reflection tracking algorithm for the SVS application to be discussed below.

The current reflected signal data collection method does not use any information from the received reflected signal to determine the parameters for data collection, but rather relies entirely on the height obtained from the position solution and the elevation contained in the DEM. This method typically insures that the onset of the reflected signal is seen in the data collected, but that onset can occur anywhere within a range of bins where data is collected. This has indeed proven to be the case in analysis of data collected from recent missions flown by the instrument. While this method of data collection is satisfactory for many applications that make use of the reflected data, it results in significant uncertainty in the measurement of the direct to reflected time difference due to the necessity to interpolate between two measurements whose positions relative to the actual time delay can only be estimated. In order to reduce this uncertainty, a feedback control algorithm is being designed that will dynamically slew the bulk delay in fine increments 
based on the characteristics of the currently measured power versus delay information in the reflected signal. This refinement in the operation of the GPS-BR receiver, along with appropriate tuning and calibration, should result in the ability to produce the direct to reflected signal time difference directly from the correlator hardware registers with accuracies approaching one half nanosecond under ideal conditions.

The development of the bulk delay tuning control algorithm is in the very early phase of design, and some research is still needed to determine all of the characteristics that need to be taken into account for its development. It is expected that the shape of the reflected signal power versus delay characteristic will have a significant influence and will likely be the most time consuming factor in the development. Early steps in the development involve performing test flights over known terrain types while slowly varying the bulk delay in order to collect the data needed to characterize the measured shapes as the bulk delay changes. Also, some experimentation will be done with the code chip spacing between reflected data samples. For GPS signals, a code chip represents 977.5 nsec. The current receiver implementation uses a spacing of one half code chip between samples, and it will be fairly easy to obtain a spacing of one quarter code chip for the experimental testing. The time difference between the direct signal channel and the reflected signal channels can then be produced to the nearest $1 / 2048^{\text {th }}$ of a chip. This corresponds to a resolution of $0.477 \mathrm{~ns}$ or $14.31 \mathrm{~cm}$. While this measurement capability indicates the theoretical potential for achieving an altitude measurement accuracy of $7.5 \mathrm{~cm}$ (one half the resolution) when tracking a high elevation satellite over level ground, it remains to be seen what actual accuracy can be achieved through tuning of the bulk delay control algorithm, and calibration of the final implemented system.

\section{SUMMARY}

By building on the previous successes of applications using the technique for measuring the GPS signal reflected from the surface of the Earth, we have shown how the concept can be applied toward the development of a SVS DEM integrity checking function thus enabling SVS to be used to its full potential. Several GPS-BR applications that are currently being used or proposed to be used were reviewed. The status of the NASA GPS-BR Receiver development was then described, and the steps being taken to enhance the capability of the receiver and enable it to provide the capability needed by the SVS system were reviewed. GPS_BR receiver technology has been demonstrated to be a promising technique for verifying synthesized terrain environments such as those used by SVS, and with the improvements envisioned herein, we intend to help that promise to become a reality.

\section{REFERENCES}

1. Bailey, R., Parrish, R., Arthur, J., and Norman, R., "Flight Test Evaluation of Tactical Synthetic Vision Display Concepts in a Terrain-Challenged Operating Environment," in Proceedings of SPIE Vol. 4713 Enhanced and Synthetic Vision 2002, edited by Jacques G. Verly, SPIE, Bellingham, WA, 2002, pp. 178-189.

2. Young, S., "On the Development of In-flight Autonomous Integrity Monitoring of Stored Geo-Spatial Data Using Forward-Looking Remote Sensing Technology,” PhD dissertation, Ohio University, March, 2005.

3. Sturtevant, P., Masters, D., Axelrad, P., and Katzberg, S., "GPS Based Bistatic Radar for Terrain Awareness Methods and Preliminary Results," Proceedings of the Institute of Navigation GPS/GNSS Technical Meeting, Portland, OR, September 9-12, 2003. 
4. Auber, Jean-Claude; Bibaut, Alain; and Rigal, Jean-Marie: Characterization of Multipath on Land and Sea at GPS Frequencies. Proceedings of $7^{\text {th }}$ International Technical Meeting of the Satellite Division of the Institute of Navigation, Part 2, ION GPS-94, Sept. 1994, pp. 1155-1171.

5. Lin, B. Katzberg, S.J., Garrison, J.L., and Wielicki, B (1999). "The Relationship Between the GPS Signals Reflected from Sea Surface and the Surface Winds: Modeling Results and Comparisons with Aircraft Measurements." Journal of Geophysical Research, Vol. 104(C9), pp. 20713-20727.

6. Katzberg, S. J., and Garrison, J. L. Jr. (1996), Utilizing GPS to determine ionospheric delay over the ocean. NASA Tech. Memo. - 4750. http://techreports.larc.nasa.gov/ltrs/PDF/NASA-96-tm4750.pdf

7. Katzberg, S. J., and Garrison, J. L.: "Wind Speed Retrieval of GPS Surface Reflection Data Using a Matched Filter Approach". Presented at the Sixth International Conference on Remote Sensing for Marine and Coastal Environments, Charleston, South Carolina, 1-3 May 2000.

8. Garrison, J.L. and Katzberg, S.J. (1998). The Application of Reflected GPS Signals to Ocean and Wetland Remote Sensing, In the Proceedings of the Fifth International Conference on Remote Sensing for Marine and Coastal Environments, San Diego, CA, 5-7 October, Vol. 1, pp. 522-529. Accepted for publication in the Journal of Remote Sensing of Environment.

9. Komjathy, Attila, Garrison, James L. and Zavorotny, Valery, "GPS A New Tool for Ocean Science”, GPS World, April 1999. 